\title{
The Maximum Surplus before Ruin in an Erlang $(n)$ Risk Process and Related Problems
}

\author{
Shuanming Li and David C.M. Dickson
}

\begin{abstract}
We study the distribution of the maximum surplus before ruin in a Sparre Andersen risk process with the inter-claim times being Erlang $(n)$ distributed. This distribution can be analyzed through the probability that the surplus process attains a given level from the initial surplus without first falling below zero. This probability, viewed as a function of the initial surplus and the given level, satisfies a homogeneous integro-differential equation with certain boundary conditions. Its solution can be expressed as a linear combination of $n$ linearly independent particular solutions of the homogeneous integrodifferential equation. Explicit results are obtained when the individual claim amounts are rationally distributed. When $n=2$, all the results can be expressed explicitly in terms of the non-ruin probability. We apply our results by looking at (i) the maximum severity of ruin and (ii) the distribution of the amount of dividends under a constant dividend barrier.
\end{abstract}

Keywords: Sparre Andersen risk model; Erlang inter-claim times; Integro-differential equation; Maximum surplus before ruin; Maximum severity of ruin; Dividends.

\section{Introduction}

Consider a Sparre Andersen surplus process

$$
U(t)=u+c t-\sum_{i=1}^{N(t)} X_{i}, \quad t \geq 0
$$

where $u \geq 0$ is the initial surplus. The $X_{i}$ 's are independent and identically distributed (i.i.d.) random variables with common distribution function $P$ and density function $p$, representing individual claim amounts. Denote by 
$\mu_{k}=E\left[X_{1}^{k}\right]$ the $k$-th moment of $X_{1}$ and by $\hat{p}(s)=\int_{0}^{\infty} e^{-s x} p(x) d x$ its Laplace transform. The counting process $\{N(t) ; t \geq 0\}$ denotes the number of claims up to time $t$ and is defined as $N(t)=\max \left\{k: W_{1}+W_{2}+\ldots+W_{k} \leq t\right\}$, where the inter-claim times $\left\{W_{i}\right\}_{i=1}^{\infty}$ are assumed to be i.i.d. random variables with common density function $k$.

Further, we assume that the sequences $\left\{W_{i}\right\}_{i=1}^{\infty}$ and $\left\{X_{i}\right\}_{i=1}^{\infty}$ are independent, and that $c E\left(W_{1}\right)>E\left(X_{1}\right)$, providing a positive safety loading factor.

For $u \geq 0$, define

$$
T=\inf \{t>0: U(t)<0\} \quad(\infty, \text { otherwise })
$$

to be the time of ruin, and $\Psi(u)=P(T<\infty)$ to be the ultimate ruin probability.

In this paper we consider the situation when the inter-claim times are Erlang distributed, a model which has been widely discussed in recent literature. See Li and Garrido (2004a) and references therein for details. We start in Section 2 by considering the maximum surplus before ruin by establishing an integro-differential equation for the probability that ruin occurs without the surplus attaining a specified level prior to ruin, and in Section 3 we apply techniques developed in $\mathrm{Li}$ and Garrido (2004b) to show how this equation can be solved. In Section 4 we show how the situation simplifies in the special case when $n=2$. We then consider two related problems: in Section 5 we consider the maximum severity of ruin, and in Section 6 we consider the distribution of the amount of dividends payable prior to ruin when there is a constant dividend barrier. In this final section we also consider the Laplace transform of the time when the surplus process reaches the dividend barrier.

\section{The maximum surplus before ruin}

For $b>u \geq 0$, define

$$
\xi(u, b)=P\left(\sup _{0 \leq t \leq T} U(t)<b, T<\infty \mid U(0)=u\right)
$$

to be the probability that ruin occurs from initial surplus $u$ without the surplus process reaching level $b$ prior to ruin. Alternatively, $\xi(u, b)$ is the probability of ruin in the presence of an absorbing barrier at $b$. Obviously, $\xi(u, b)=0$ for $b \leq u$.

We now show that $\xi(u, b)$ satisfies an integro-differential equation with certain boundary conditions. 
Theorem 2.1 If there exists a positive integer $L$, such that the density function, $k$, of the inter-claim times for the risk model (1.1) has the property

$$
k^{(l)}(0)=0, \quad l=0,1, \ldots, L-1,
$$

with $k^{(0)}=k$, then $\xi(u, b)$ satisfies the boundary conditions

$$
\left.\frac{\partial^{l} \xi(u, b)}{\partial u^{l}}\right|_{u=b}=0, \quad l=0,1,2, \ldots, L
$$

with $\partial^{0} \xi(u, b) /\left(\partial u^{0}\right)=\xi(u, b)$.

Proof: Conditioning on the time and the amount of the first claim, we have

$$
\xi(u, b)=\int_{0}^{(b-u) / c} k(t) \gamma_{b}(u+c t) d t
$$

for $0 \leq u<b$, where $\gamma_{b}(t)=\int_{0}^{t} \xi(t-x, b) p(x) d x+\bar{P}(t)$, and $\bar{P}=1-P$. Substituting $s=u+c t$, we obtain

$$
\xi(u, b)=\frac{1}{c} \int_{u}^{b} k\left(\frac{s-u}{c}\right) \gamma_{b}(s) d s .
$$

The boundary condition (2.2) for $l=0$ is obvious. For $l=1,2, \ldots, L$, taking the $l$-th derivative of both sides of (2.3) with respect to $u$ and using the conditions in (2.1) yields

$$
\frac{\partial^{l} \xi(u, b)}{\partial u^{l}}=\frac{(-1)^{l}}{c^{l+1}} \int_{u}^{b} k^{(l)}\left(\frac{s-u}{c}\right) \gamma_{b}(s) d s .
$$

Setting $u=b$ in (2.4) shows that the boundary conditions (2.2) hold for $l=1,2, \ldots, L$. This completes the proof.

For the rest of this paper, we assume that the inter-claim times are $\operatorname{Erlang}(n)$ distributed with scale parameter $\lambda>0$. We use $k_{n}$ to denote their common density function, which is given by

$$
k_{n}(t ; \lambda)=\frac{\lambda^{n} t^{n-1} e^{-\lambda t}}{(n-1) !}, \quad t \geq 0, n \in \mathbb{N}^{+} .
$$

We have the following result for $\xi(u, b)$.

Theorem 2.2 If the inter-claim times are Erlang $(n)$ distributed with density function given by (2.5), then $\xi(u, b)$ satisfies the integro-differential equation

$$
\sum_{k=0}^{n} \frac{\partial^{k} \xi(u, b)}{\partial u^{k}}\left(\frac{-c}{\lambda}\right)^{k}\left(\begin{array}{c}
n \\
n-k
\end{array}\right)=\int_{0}^{u} \xi(u-y, x) p(y) d y+\bar{P}(u)
$$


for $0 \leq u<b$, with boundary conditions

$$
\left.\frac{\partial^{k} \xi(u, b)}{\partial u^{k}}\right|_{u=b}=0, \quad k=0,1,2, \ldots, n-1
$$

Proof: Since the density function $k_{n}$ in (2.5) has the property that

$$
k_{n}^{(l)}(0 ; \lambda)=0, \quad l=0,1,2, \ldots, n-2, \quad n \geq 2,
$$

$k_{n}$ satisfies condition (2.1) in Theorem 2.1 for $L=n-1$. Therefore, the boundary conditions in (2.7) hold true.

For $j=1,2, \ldots, n-1$, applying the operator $[\partial / \partial u-(\lambda / c) \mathcal{I}]^{j}$ to both sides of $(2.3)$ and noting that $k_{n}^{\prime}(t ; \lambda)=\lambda\left[k_{n-1}(t ; \lambda)-k_{n}(t ; \lambda)\right]$ gives

$$
\left[\frac{\partial}{\partial u}-\left(\frac{\lambda}{c}\right) \mathcal{I}\right]^{j} \xi(u, b)=\frac{(-\lambda)^{j}}{c^{j+1}} \int_{u}^{b} k_{n-j}\left(\frac{s-u}{c} ; \lambda\right) \gamma_{b}(s) d s
$$

where $\mathcal{I}$ denotes the $i d e n t i t y$ operator. In particular, when $j=n-1$,

$$
\left[\frac{\partial}{\partial u}-\left(\frac{\lambda}{c}\right) \mathcal{I}\right]^{n-1} \xi(u, b)=\frac{(-\lambda)^{n-1}}{c^{n}} \int_{u}^{b} k_{1}\left(\frac{s-u}{c} ; \lambda\right) \gamma_{b}(s) d s
$$

Applying the operator $[\partial / \partial u-(\lambda / c) \mathcal{I}]$ to both sides of $(2.8)$ and noting that $\gamma_{b}(u)=\int_{0}^{u} \xi(u-x, b) p(x) d x+\bar{P}(u)$, we have

$$
\left[\frac{\partial}{\partial u}-\left(\frac{\lambda}{c}\right) \mathcal{I}\right]^{n} \xi(u, b)=\left(\frac{-\lambda}{c}\right)^{n}\left[\int_{0}^{u} \xi(u-x, b) p(x) d x+\vec{P}(u)\right]
$$

which is equivalent to (2.6) after dividing both sides by $(-\lambda / c)^{n}$.

Next, define

$$
\tau^{b}=\inf \{t>0: U(t) \geq b \mid U(0)=u\}, \quad 0 \leq u \leq b
$$

to be the first time that the surplus process upcrosses the level $b$, and

$$
\chi(u, b)=P\left(T>\tau^{b} \mid U(0)=u\right)
$$

to be the probability that the surplus process attains a given level $b$ from initial surplus $u$ without first falling below zero. Since eventually either ruin occurs without the surplus process attaining level $b$ or the surplus attains level $b$, then we have

$$
\chi(u, b)=1-\xi(u, b)
$$


Corollary 2.3 The probability $\chi(u, b)$ satisfies the homogeneous integrodifferential equation

$$
\sum_{k=0}^{n} \frac{\partial^{k} \chi(u, b)}{\partial u^{k}}\left(\frac{-c}{\lambda}\right)^{k}\left(\begin{array}{c}
n \\
n-k
\end{array}\right)=\int_{0}^{u} \chi(u-y, b) p(y) d y
$$

for $0 \leq u<b$, with boundary conditions

$$
\begin{aligned}
\chi(b, b) & =1, \\
\left.\frac{\partial^{k} \chi(u, b)}{\partial u^{k}}\right|_{u=b} & =0, \quad k=1,2, \ldots, n-1 .
\end{aligned}
$$

Proof: This easily follows from Theorem 2.2 by noting that $\chi(u, b)=$ $1-\xi(u, b)$.

\section{The solution of an integro-differential equa- tion}

In this section, we consider the solution of the homogenous integro-differential equation

$$
B(\mathcal{D}) v(u)=\int_{0}^{u} v(u-y) p(y) d y, \quad u \geq 0
$$

with boundary conditions

$$
v^{(k)}(b)=e_{k}, \quad k=0,1,2, \ldots, n-1,
$$

where $e_{1}, e_{2}, \ldots, e_{n}$ are constants, $\mathcal{D}$ denotes the differentiation operator and

$$
B(\mathcal{D})=[\mathcal{I}-(c / \lambda) \mathcal{D}]^{n}=\sum_{k=0}^{n}(-1)^{k}\left(\frac{c}{\lambda}\right)^{k}\left(\begin{array}{l}
n \\
k
\end{array}\right) \mathcal{D}^{k}=\sum_{k=0}^{n} B_{k} \mathcal{D}^{k}
$$

is an $n$-th order linear differentiation operator.

It follows from the theory of differential equations that the solution of equation (3.1) with boundary conditions (3.2) is of the form

$$
v(u)=\sum_{i=1}^{n} \eta_{i} v_{i}(u), \quad u \geq 0
$$

where $v_{i}(u), i=1,2, \ldots, n$, are $n$ linearly independent particular solutions of equation (3.1), and $\eta_{1}, \eta_{2}, \ldots, \eta_{n}$ are determined by the following system of linear equations

$$
\sum_{i=1}^{n} \eta_{i} v_{i}^{(k)}(b)=e_{k}, \quad k=0,1,2, \ldots, n-1
$$


Dickson (1998) shows that $\Phi(u)=1-\Psi(u)$ satisfies the integro-differential equation (3.1). Thus we can employ $v_{1}(u)=\Phi(u)$ as a particular solution.

One can find the $n-1$ linearly independent solutions $v_{i}(u), i=2,3, \ldots, n$, by specifying the initial conditions by

$$
v_{i}^{(k)}(0)=I(k=i-1), \quad k=0,1, \ldots, n-1,
$$

where $I(\cdot)$ denotes the indicator function. To prove that $v_{i}(u), i=1,2, \ldots, n$, are linearly independent, we assume there are constants $c_{1}, c_{2}, \ldots, c_{n}$ such that $\sum_{i=1}^{n} c_{i} v_{i}(u) \equiv 0$, for any $u \geq 0$. Then $\sum_{i=1}^{n} c_{i} v_{i}^{(k)}(u) \equiv 0$, for $k=$ $0,1, \ldots, n-1$, and any $u \geq 0$. Setting $u=0$ and noting that $v_{1}(0)=\Phi(0) \neq 0$ and $v_{i}^{(k)}(0)=I\{k=i-1\}$, for $k=0,1,2, \ldots, n-1$, we can prove that $c_{1}=c_{2}=\cdots=c_{n}=0$.

From Section 6 of Li and Garrido (2004a) it is straightforward to show that $\Phi(u)$ satisfies the defective renewal equation

$$
\Phi(u)=\int_{0}^{u} \Phi(u-y) g(y) d y+\frac{\lambda^{n} \theta \mu_{1}}{c^{n} \rho_{1} \rho_{2} \cdots \rho_{n-1}}, \quad u \geq 0,
$$

where $\theta=c n /\left(\lambda \mu_{1}\right)-1$ is the relative safety loading factor, $\rho_{1}, \rho_{2}, \ldots, \rho_{n-1}$, with $\Re\left(\rho_{i}\right)>0$, are the roots of the generalized Lundberg equation $B(s)=$ $\hat{p}(s)$ in the right half complex plane, and

$$
g(y)=\frac{\lambda^{n}}{c^{n}} T_{0} T_{\rho_{1}} \cdots T_{\rho_{n-1}} p(y),
$$

with $T_{r}$ being an operator, such that

$$
T_{r} f(x)=\int_{x}^{\infty} e^{-r(y-x)} f(y) d y, \quad r \in \mathbb{C},
$$

for any integrable real function $f$.

$\mathrm{Li}$ and Garrido (2004b) show that $v_{i}(u), i=2,3, \ldots, n$, all satisfy the defective renewal equation

$$
v_{i}(u)=\int_{0}^{u} v_{i}(u-y) g(y) d y+\xi_{i, 0}+\sum_{j=1}^{n-1} \xi_{i, j} e^{\rho_{j} u}, \quad u \geq 0
$$

where

$$
\begin{aligned}
\xi_{i, 0} & =-\frac{\lambda^{n} B_{i}}{c^{n} \rho_{1} \rho_{2} \cdots \rho_{n-1}}, \\
\xi_{i, j} & =\frac{\lambda^{n} \sum_{m=0}^{n-i} B_{m+i} \rho_{j}^{m}}{c^{n} \rho_{j} \prod_{k=1, k \neq j}^{n-1}\left(\rho_{k}-\rho_{j}\right)}, \quad j=1,2, \ldots, n-1 .
\end{aligned}
$$


Further if the individual claim amounts are rationally distributed, then the Laplace transform of $v_{i}(u)$ can be inverted by partial fractions. Let us now assume that individual claim amounts are rationally distributed, i.e.,

$$
\hat{p}(s)=\frac{Q_{m-1}(s)}{Q_{m}(s)}, \quad \Re(s) \in\left(h_{X}, \infty\right),
$$

where $m \in \mathbb{N}^{+}, h_{X}:=\inf \left\{s \in R: E\left[e^{-s X}\right]<\infty\right\}, Q_{m}$ is a polynomial of degree $m$ with leading coefficient $1, Q_{m-1}$ is a polynomial of degree $m-1$ or less, and $Q_{m}$ and $Q_{m-1}$ do not have any common zeros. Further, since $\hat{p}(s)$ is finite for all $s$, with $\Re(s)>0$, the equation $Q_{m}(s)=0$ has no roots with negative real parts.

Let $\rho_{1}, \rho_{2}, \ldots, \rho_{n-1}$, with $\Re\left(\rho_{i}\right)>0, \rho_{n}=0$, and $-R_{1},-R_{2}, \ldots,-R_{m}$ with $\Re\left(R_{i}\right)>0$, be the roots of the equation

$$
B(s) Q_{m}(s)-Q_{m-1}(s)=[1-(c / \lambda) s]^{n} Q_{m}(s)-Q_{m-1}(s)=0 .
$$

$\mathrm{Li}$ and Garrido (2004a, eq. 20) show that

$$
\hat{v}_{1}(s)=\hat{\Phi}(s)=\frac{\Phi(0) Q_{m}(s)}{s \prod_{i=1}^{m}\left(s+R_{i}\right)} .
$$

If $R_{1}, R_{2}, \ldots, R_{m}$ are distinct, then inversion of (3.8) yields

$$
\Phi(u)=1-\Phi(0) \sum_{i=1}^{m}\left[\frac{Q_{m}\left(-R_{i}\right)}{R_{i} \prod_{j=1, j \neq i}^{m}\left(R_{j}-R_{i}\right)}\right] e^{-R_{i} u}, \quad u \geq 0 .
$$

$\mathrm{Li}$ and Garrido (2004b) show that the Laplace transform of $v_{i}(u), i=2,3, \ldots, n$, can be expressed as

$$
\hat{v}_{i}(s)=\frac{\lambda^{n}}{c^{n}} \frac{\left[-d_{i}(s) Q_{m}(s)\right]}{s\left[\prod_{j=1}^{n-1}\left(\rho_{j}-s\right)\right]\left[\prod_{j=1}^{m}\left(s+R_{j}\right)\right]},
$$

where $d_{i}(s)=\sum_{k=i}^{n} B_{k} s^{k-i}=\sum_{l=0}^{n-i} B_{l+i} s^{l}$.

If $\rho_{1}, \rho_{2}, \ldots, \rho_{n-1}$ and $R_{1}, R_{2}, \ldots, R_{m}$ are all distinct, by partial fractions, we have

$$
\hat{v}_{i}(s)=\frac{\alpha_{i, 0}}{s}+\sum_{k=1}^{n-1} \frac{\alpha_{i, k}}{\left(s-\rho_{k}\right)}+\sum_{j=1}^{m} \frac{\beta_{i, j}}{\left(s+R_{j}\right)},
$$

where

$$
\begin{aligned}
\alpha_{i, 0} & =-\frac{\lambda^{n} B_{i} Q_{m}(0)}{c^{n}\left[\prod_{l=1}^{n-1} \rho_{i}\right]\left[\prod_{j=1}^{m} R_{j}\right]} \\
\alpha_{i, k} & =-\frac{\lambda^{n} d_{i}\left(\rho_{k}\right) Q_{m}\left(\rho_{k}\right)}{c^{n} \prod_{j=1}^{m}\left(R_{j}+\rho_{k}\right) \prod_{l=1, l \neq k}^{n}\left(\rho_{l}-\rho_{k}\right)} \\
\beta_{i, j} & =\frac{\lambda^{n} d_{i}\left(-R_{j}\right) Q_{m}\left(-R_{j}\right)}{c^{n} \prod_{l=1}^{n}\left(R_{j}+\rho_{l}\right) \prod_{r=1, r \neq j}^{m}\left(R_{r}-R_{j}\right)}
\end{aligned}
$$


Then inverting (3.11) yields

$$
v_{i}(u)=\alpha_{i, 0}+\sum_{k=1}^{n} \alpha_{i, k} e^{\rho_{k} u}+\sum_{j=1}^{m} \beta_{i, j} e^{-R_{j} u}, \quad i=2,3, \ldots, n, u \geq 0
$$

\section{Analysis of $\chi(u, b)$ when $n=2$}

In this section, we give a detailed discussion of the results for the risk model with Erlang(2) inter-claim times. Dickson and Hipp (1998) show that

$$
\hat{\Phi}(s)=\Phi(0) \frac{c^{2}(s-\rho)}{\lambda^{2}[B(s)-\hat{p}(s)]},
$$

where $\rho>0$ is the unique positive root of the equation $B(s)=\hat{p}(s)$. Li and Garrido (2004b) show that

$$
\hat{v}_{2}(s)=\frac{c^{2}}{\lambda^{2}[B(s)-\hat{p}(s)]} .
$$

By comparing these two Laplace transforms we obtain

$$
v_{2}(u)=\frac{\int_{0}^{u} \Phi(u-y) e^{\rho y} d y}{\Phi(0)}
$$

Thus, $\chi(u, b)$ can be expressed explicitly in terms of the non-ruin probability $\Phi(u)$. Equation (3.3) shows that $\chi(u, b)$ is of the form

$$
\chi(u, b)=\eta_{1}(b) \Phi(u)+\eta_{2}(b) \frac{\int_{0}^{u} \Phi(u-y) e^{\rho y} d y}{\Phi(0)}
$$

where $\eta_{1}(b)$ and $\eta_{2}(b)$ are determined by the boundary conditions (2.9) and (2.10), which can be solved to obtain

$$
\begin{aligned}
& \eta_{1}(b)=\frac{\Phi(b)+\rho \int_{0}^{b} \Phi(b-y) e^{\rho y} d y}{\Phi^{2}(b)+\left[\rho \Phi(b)-\Phi^{\prime}(b)\right] \int_{0}^{b} \Phi(b-y) e^{\rho y} d y} \\
& \eta_{2}(b)=\frac{-\Phi(0) \Phi^{\prime}(b)}{\Phi^{2}(b)+\left[\rho \Phi(b)-\Phi^{\prime}(b)\right] \int_{0}^{b} \Phi(b-y) e^{\rho y} d y}
\end{aligned}
$$

We remark that when individual claim amounts are rationally distributed, a closed form solution exists for $\Phi(u)$, and hence for $\chi(u, b)$. It is interesting that, just as in the classical risk model, only a knowledge of $\Phi(u)$ is required to find $\chi(u, b)$. 


\section{The maximum severity of ruin}

In this section we allow the surplus process to continue if ruin occurs, and consider the insurer's maximum severity of ruin from the time of ruin until the time that the surplus returns to level 0 . Since we assume that $c n>\lambda \mu_{1}$, it is certain that the surplus process will attain this level.

Following Picard (1994), for $u \geq 0$ we define $T^{\prime}$ to be the time of the first upcrossing of the surplus process through level 0 after ruin occurs, i.e.,

$$
T^{\prime}=\inf \{t: t>T, U(t) \geq 0\},
$$

and define

$$
M_{u}=\sup \left\{|U(t)|, T \leq t \leq T^{\prime}\right\}
$$

Let

$$
J(z ; u)=P\left(M_{u} \leq z \mid T<\infty\right), \quad z \geq 0,
$$

denote the distribution function of the maximum severity of ruin given that ruin occurs.

As shown in Dickson (1998),

$$
J(z ; u)=\frac{1}{\Psi(u)} \int_{0}^{z} g(u, y) \chi(z-y, z) d y,
$$

where $g(u, y)=\partial G(u, y) / \partial y$, with

$$
G(u, y)=P(T<\infty, U(T) \geq-y)
$$

being the probability that ruin occurs and that the deficit at ruin is at most $y$.

We now consider equation (5.1) when $n=2$. We have

$$
\begin{aligned}
J(z ; u)= & \frac{1}{\Psi(u)} \int_{0}^{z} g(u, y) \chi(z-y, z) d y \\
= & \frac{\eta_{1}(z)}{\Psi(u)} \int_{0}^{z} g(u, y) \Phi(z-y) d y \\
& +\frac{\eta_{2}(z)}{\Phi(0) \Psi(u)} \int_{0}^{z} g(u, y) \int_{0}^{z-y} \Phi(z-y-x) e^{\rho x} d x d y .
\end{aligned}
$$

Noting that

$$
\Psi(u+z)=\int_{z}^{\infty} g(u, y) d y+\int_{0}^{z} g(u, y) \Psi(z-y) d y,
$$


and

$$
\int_{0}^{z} g(u, y) \Phi(z-y) d y=\int_{z}^{\infty} g(u, y) d y+\int_{0}^{z} g(u, y) d y-\Psi(u+z)=\Psi(u)-\Psi(u+z),
$$

we have

$$
\begin{array}{rl}
\int_{0}^{z} & g(u, y) \int_{0}^{z-y} \Phi(z-y-x) e^{\rho x} d x d y \\
& =\int_{0}^{z} g(u, z-y) \int_{0}^{y} \Phi(y-x) e^{\rho x} d x d y \\
& =\int_{0}^{z} e^{\rho x} \int_{x}^{z} g(u, z-y) \Phi(y-x) d y d x \\
& =\int_{0}^{z} e^{\rho x} \int_{0}^{z-x} g(u, t) \Phi(z-x-t) d t d x \\
& =\int_{0}^{z} e^{\rho x}[\Psi(u)-\Psi(u+z-x)] d x \\
& =\int_{0}^{z} e^{\rho x}[\Phi(u+z-x)-\Phi(u)] d x
\end{array}
$$

Finally, we have

$$
\begin{aligned}
J(z ; u)= & \frac{\Phi(z)+\rho \int_{0}^{z} \Phi(z-y) e^{\rho y} d y}{\Phi^{2}(z)+\left[\rho \Phi(z)-\Phi^{\prime}(z)\right] \int_{0}^{z} \Phi(z-y) e^{\rho y} d y}\left[\frac{\Phi(u+z)-\Phi(u)}{1-\Phi(u)}\right] \\
& -\frac{\Phi^{\prime}(z) \int_{0}^{z} e^{\rho x}[\Phi(u+z-x)-\Phi(u)] d x}{\Phi^{2}(z)+\left[\rho \Phi(z)-\Phi^{\prime}(z)\right] \int_{0}^{z} \Phi(z-y) e^{\rho y} d y} \frac{1}{1-\Phi(u)}
\end{aligned}
$$

As in the previous section, when individual claim amounts are rationally distributed, a closed form solution exists for $\Phi(u)$, and hence for $J(z ; u)$.

\section{Dividends problems}

In this section we consider the situation when there is a constant dividend barrier at $b \geq u$. We assume that whenever the surplus reaches level $b$ that premium income is paid out to shareholders as dividends. The surplus process thus remains at level $b$ until a claim occurs, and on any subsequent return to level $b$, premium income is again paid out as dividends. For this modified surplus process, it is straightforward to show that ultimate ruin is certain. 


\subsection{The distribution of the amount of dividends}

Dickson and Waters (2004) consider the distribution of the total amount of dividends payable until ruin in the classical risk model. We now extend their arguments to the $\operatorname{Erlang}(n)$ risk model. Let $D_{u}$ denote the total amount of dividends payable until ruin. Then

$$
D_{u}=\sum_{i=1}^{N} \Delta_{i}
$$

where $N$ denotes the number of streams of dividend payments, and $\Delta_{i}$ denotes the amount of dividends in the $i$-th dividend stream. Then $N$ has a zero-modified geometric distribution, with $P(N=0)=1-\chi(u, b)$ and

$$
P(N=r)=\chi(u, b)[1-a(b)] a(b)^{r-1}, \quad r=1,2, \ldots,
$$

where $a(b)=\int_{0}^{b} \chi(b-x, b) p(x) d x$. Further, $\left\{\Delta_{i}\right\}_{i=1}^{\infty}$ is a sequence of i.i.d. random variables with density function

$$
f_{\Delta}(x)=\sum_{i=1}^{n} \frac{\chi_{i}(u, b)}{\chi(u, b)} k_{n+1-i}(x ; \lambda / c)
$$

where $k_{n+1-i}(x ; \lambda / c)$ is the $\operatorname{Erlang}(n+1-i)$ density function with scale parameter $\lambda / c$, and $\chi_{i}(u, b)$ is the probability that the surplus reaches the dividend barrier $b$ during phase $i$ (explained below) from initial surplus $u$, for $i=1,2, \ldots, n$, so that

$$
\chi(u, b)=\sum_{i=1}^{n} \chi_{i}(u, b)
$$

Motivated by Gerber and Shiu (2005), we decompose the Erlang $(n)$ interclaim time $W_{1}$ into $n$ phases as

$$
W_{1}=V_{1}+V_{2}+\ldots+V_{n}
$$

where the $V_{i}$ 's are independent, exponentially distributed random variables with parameter $\lambda>0$. Thus, if the surplus reaches $b$ during the first phase, the distribution of the time until the end of that phase is exponential with parameter $\lambda$, and hence the distribution of the time until the first claim is still $\operatorname{Erlang}(n)$ with scale parameter $\lambda$. Similarly, if the surplus reaches $b$ during the second phase, the distribution of the time until the end of that phase is exponential with parameter $\lambda$, and hence the distribution of the time until 
the first claim is Erlang $(n-1)$ with scale parameter $\lambda$. The same argument applies to the other phases, and the other claim inter-arrival times.

Conditioning on whether the surplus reaches the barrier $b$ before or after the first claim, we have for $i=1,2, \ldots, n$,

$$
\begin{aligned}
& \chi_{i}(u, b) \\
= & P\left(\sum_{j=1}^{i-1} V_{j} \leq \frac{b-u}{c}<\sum_{j=1}^{i} V_{j}\right)+\int_{0}^{(b-u) / c} k_{n}(t ; \lambda) \gamma_{i}(u+c t, b) d t \\
= & \bar{K}_{i}\left(\frac{b-u}{c} ; \lambda\right)-\bar{K}_{i-1}\left(\frac{b-u}{c} ; \lambda\right)+\frac{1}{c} \int_{u}^{b} k_{n}\left(\frac{s-u}{c} ; \lambda\right) \gamma_{i}(s, b) d s,
\end{aligned}
$$

where $\gamma_{i}(z, b)=\int_{0}^{z} \chi_{i}(z-x, b) p(x) d x$ and $\bar{K}_{i}(t ; \lambda)$ is the survival function of the Erlang $(i)$ distribution with scale parameter $\lambda$, with $\bar{K}_{0}(t ; \lambda)=0$ for all $t$. It is well known that

$$
\bar{K}_{i}(t ; \lambda)=\sum_{j=0}^{i-1} \frac{(\lambda t)^{j} e^{-\lambda t}}{j !}, \quad t \geq 0, i=1,2, \ldots, n
$$

Thus, (6.3) can be rewritten as

$$
\chi_{i}(u, b)=\left(\frac{-\lambda}{c}\right)^{i-1} \frac{(u-b)^{i-1}}{(i-1) !} e^{-\lambda(b-u) / c}+\frac{1}{c} \int_{u}^{b} k_{n}\left(\frac{s-u}{c} ; \lambda\right) \gamma_{i}(s, b) d s
$$

for $i=1,2, \ldots, n$. Then we have the following results.

Theorem 6.1 For $i=1,2, \ldots, n$, the function $\chi_{i}(u, b)$ satisfies the boundary conditions

$$
\left.\frac{\partial^{l} \chi_{i}(u, b)}{\partial u^{l}}\right|_{u=b}=(-1)^{i-1}\left(\begin{array}{c}
l \\
i-1
\end{array}\right)\left(\frac{\lambda}{c}\right)^{l} I(i-1 \leq l \leq n-1)
$$

for $l=0,1, \ldots, n-1$, with $\partial^{0} \chi_{i}(u, b) / \partial u^{0}=\chi_{i}(u, b)$.

Proof: For $l=1,2, \ldots, n-1$, taking the $l$-th derivative of both sides of (6.3) with respect to $u$, we have

$$
\begin{aligned}
\frac{\partial^{l} \chi_{i}(u, b)}{\partial u^{l}}= & \left(\frac{-\lambda}{c}\right)^{i-1} \frac{\partial^{l}}{\partial u^{l}}\left[\frac{(u-b)^{i-1}}{(i-1) !} e^{-\lambda(b-u) / c}\right] \\
& +\frac{(-1)^{l}}{c^{d+1}} \int_{u}^{b} k_{n}^{(l)}\left(\frac{s-u}{c} ; \lambda\right) \gamma_{i}(s, b) d s
\end{aligned}
$$


Setting $u=b$ in the above equation yields the boundary condition (6.4), and setting $u=b$ in (6.3) gives

$$
\chi_{i}(b, b)=I(i=1)
$$

which can be rewritten as (6.4) for $l=0$.

Theorem 6.2 For $i=1,2, \ldots, n$, and $0 \leq u<b$, the function $\chi_{i}(u, b)$ satisfies the integro-differential equation

$$
\sum_{k=0}^{n} \frac{\partial^{k} \chi_{i}(u, b)}{\partial u^{k}}\left(\frac{-c}{\lambda}\right)^{k}\left(\begin{array}{c}
n \\
n-k
\end{array}\right)=\int_{0}^{u} \chi_{i}(u-y, b) p(y) d y
$$

with boundary conditions being given by Theorem 6.1 .

Proof: For $j=1,2, \ldots, n-1$, applying the operator $[\partial / \partial u-(\lambda / c) \mathcal{I}]^{j}$ to both sides of $(6.3)$ and noting that $k_{n}^{\prime}(t ; \lambda)=\lambda\left[k_{n-1}(t ; \lambda)-k_{n}(t ; \lambda)\right]$ gives

$$
\begin{aligned}
{\left[\frac{\partial}{\partial u}-\left(\frac{\lambda}{c}\right) \mathcal{I}\right]^{j} \chi_{i}(u, b)=} & \left(\frac{-\lambda}{c}\right)^{i-1} \frac{(u-b)^{i-1-j}}{(i-1-j) !} I(j \leq i-1) e^{-\lambda(b-u) / c} \\
& +\frac{(-\lambda)^{j}}{c^{j+1}} \int_{u}^{b} k_{n-j}\left(\frac{s-u}{c} ; \lambda\right) \gamma_{i}(s, b) d s .
\end{aligned}
$$

In particular, for $i=1,2, \ldots, n-1$ and $j=n-1$,

$$
\left[\frac{\partial}{\partial u}-\left(\frac{\lambda}{c}\right) \mathcal{I}\right]^{n-1} \chi_{i}(u, b)=\frac{(-\lambda)^{n-1}}{c^{n}} \int_{u}^{b} \lambda e^{-\lambda(s-u) / c} \gamma_{i}(s, b) d s
$$

while for $i=n$ and $j=n-1$,

$$
\begin{aligned}
& {\left[\frac{\partial}{\partial u}-\left(\frac{\lambda}{c}\right) \mathcal{I}\right]^{n-1} \chi_{n}(u, b)} \\
& \quad=\left(\frac{-\lambda}{c}\right)^{n-1} e^{-\lambda(b-u) / c}+\frac{(-\lambda)^{n-1}}{c^{n}} \int_{u}^{b} \lambda e^{-\lambda(s-u) / c} \gamma_{n}(s, b) d s .
\end{aligned}
$$

Applying the operator $[\partial / \partial u-(\lambda / c) \mathcal{I}]$ again to both sides of equations (6.6) and (6.7) yields for $i=1,2, \ldots, n$,

$$
\left[\frac{\partial}{\partial u}-\left(\frac{\lambda}{c}\right) \mathcal{I}\right]^{n} \chi_{i}(u, b)=\left(\frac{-\lambda}{c}\right)^{n} \gamma_{i}(u, b) .
$$

Dividing both sides by $(-\lambda / c)^{n}$ and noting that $\gamma_{i}(u, b)=\int_{0}^{u} \chi_{i}(u-x, b) p(x) d x$, proves that the integro-differential (6.5) holds. 
Example 6.1 In this example we illustrate the weight

$$
w(u, b)=\chi_{1}(u, b) / \chi(u, b)
$$

in formula (6.2) in the special case when $n=2$ and $p(x)=e^{-x}, x>0$. Following the analysis in Section 4, we have

$$
\chi_{i}(u, b)=\eta_{i, 1}(b) \Phi(u)+\eta_{i, 2}(b) v_{2}(u)
$$

for $i=1,2$, where $v_{2}(u)$ is given by formula (4.1). The functions $\eta_{i, j}(b)$, $i, j=1,2$, can be found from the boundary conditions (6.4), yielding

$$
\eta_{1,1}(b)=\frac{c v_{2}^{\prime}(b)-\lambda v_{2}(b)}{c\left(v_{2}^{\prime}(b) \Phi(b)-v_{2}(b) \Phi^{\prime}(b)\right)}, \quad \eta_{1,2}(b)=\frac{-c \Phi^{\prime}(b)+\lambda \Phi(b)}{c\left(v_{2}^{\prime}(b) \Phi(b)-v_{2}(b) \Phi^{\prime}(b)\right)}
$$

and

$$
\eta_{2,1}(b)=\frac{\lambda v_{2}(b)}{c\left(v_{2}^{\prime}(b) \Phi(b)-v_{2}(b) \Phi^{\prime}(b)\right)}, \quad \eta_{2,2}(b)=\frac{-\lambda \Phi(b)}{c\left(v_{2}^{\prime}(b) \Phi(b)-v_{2}(b) \Phi^{\prime}(b)\right)} .
$$

It is well known (see, for example, Grandell (1991)) that

$$
\Phi(u)=1-(1-R) e^{-R u},
$$

where $R$ is the adjustment coefficient, leading to

$$
v_{2}(u)=\frac{1}{R \rho}\left(e^{\rho u}-1\right)-\frac{1-R}{R(\rho+R)}\left(e^{\rho u}-e^{-R u}\right) .
$$

Figure 6.1 shows $w(u, 10)$ for $0 \leq u \leq 10$ when $\lambda=2$ and $c=1.2$, while Figure 6.2 shows $w(u, 100)$ for $0 \leq u \leq 100$ when $\lambda=2$ and $c=1.1$. We see in each case that the weight $w(u, b)$ changes only as $u$ approaches $b$ as we might have anticipated.

\subsection{The time to reach the dividend barrier}

We now consider how long it takes for the surplus process to reach the dividend barrier $b$ from initial surplus $u(<b)$ without ruin occuring. We define $\tau_{b}$ to be the first time that the surplus reaches $b>u$ without ruin having previously occurred, and, for $\delta>0$, define

$$
\mathcal{L}(u, b)=E\left[e^{-\delta \tau_{b}} \mid U(0)=u\right], \quad 0 \leq u \leq b .
$$

Thus, $\mathcal{L}(u, b)$ can be viewed as the expected present value of one dollar payable at time $\tau_{b}$, or, alternatively, it can be viewed as the Laplace transform of $\tau_{b}$ with respect to parameter $\delta$. 


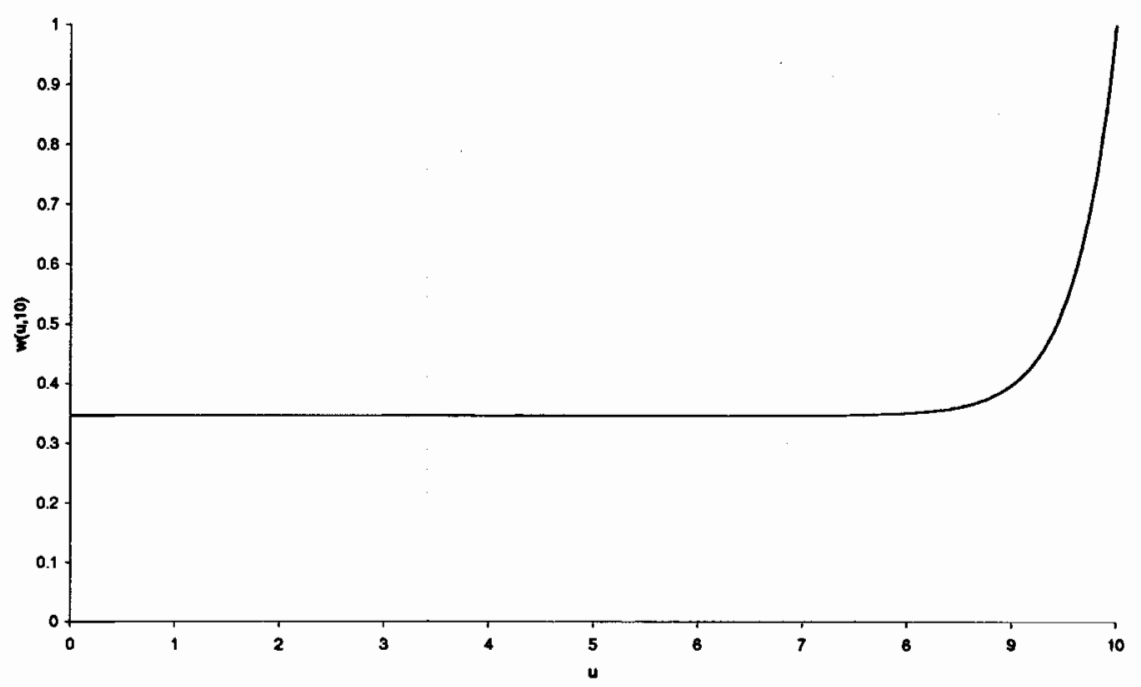

Figure 6.1: Values of $w(u, 10)$ when $c=1.2$.

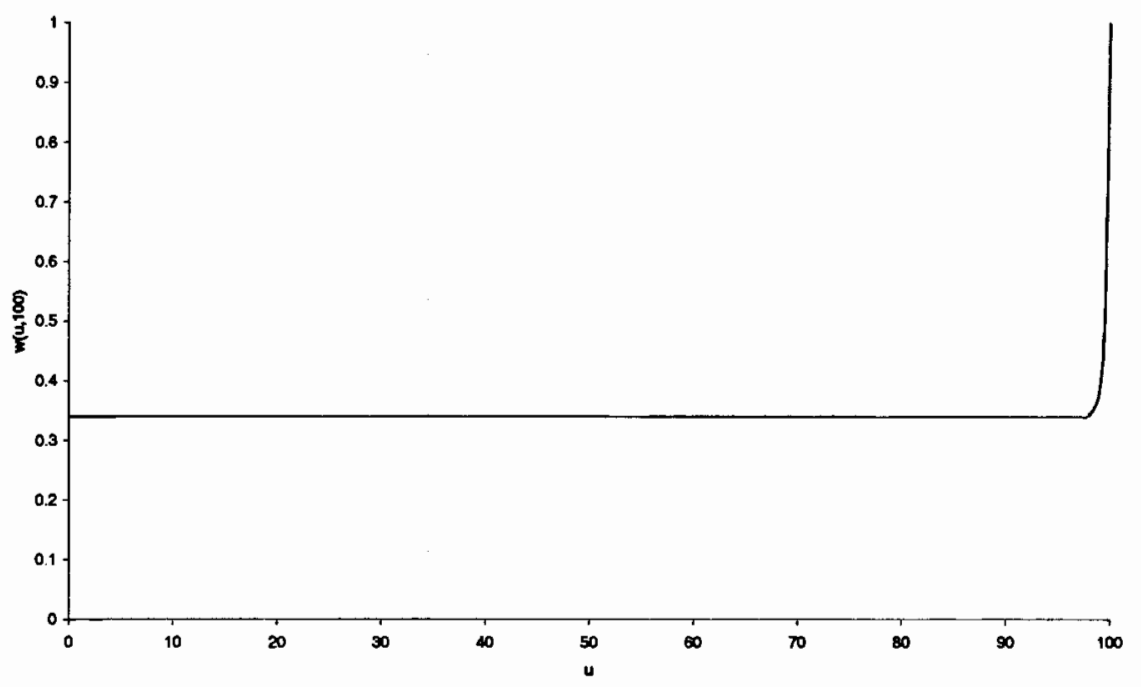

Figure 6.2: Values of $w(u, 100)$ when $c=1.1$. 
Theorem 6.3 $\mathcal{L}(u, b)$ satisfies the boundary conditions

$$
\left.\frac{\partial^{l} \mathcal{L}(u, b)}{\partial u^{l}}\right|_{u=b}=\left(\frac{\delta}{c}\right)^{l}, \quad l=0,1,2, \ldots, n-1 .
$$

Proof: Conditioning on the time and the amount of the first claim, for $0 \leq u \leq b$ we have

$$
\mathcal{L}(u, b)=\int_{0}^{(b-u) / c} e^{-\delta t} k_{n}(t ; \lambda) \zeta_{b}(u+c t) d t+e^{-\delta(b-u) / c} \bar{K}_{n}\left(\frac{b-u}{c} ; \lambda\right)
$$

where $\zeta_{b}(t)=\int_{0}^{t} \mathcal{L}(t-x, b) p(x) d x$. Substituting $s=u+c t$, we obtain

$$
\begin{aligned}
& \mathcal{L}(u, b) \\
= & \frac{1}{c} \int_{u}^{b} k_{n}\left(\frac{s-u}{c} ; \lambda\right) e^{-\delta(s-u) / c} \zeta_{b}(s) d s+e^{-\delta(b-u) / c} \bar{K}_{n}\left(\frac{b-u}{c} ; \lambda\right) \\
= & \frac{1}{c}\left(\frac{\lambda}{\lambda+\delta}\right)^{n} \int_{u}^{b} k_{n}\left(\frac{s-u}{c} ; \lambda+\delta\right) \zeta_{b}(s) d s+e^{-\delta(b-u) / c} \bar{K}_{n}\left(\frac{b-u}{c} ; \lambda\right) .
\end{aligned}
$$

Setting $u=b$ in (6.9) gives $\mathcal{L}(b, b)=1$. We know that for $n \geq 2$,

$$
\begin{aligned}
k_{n}^{(l)}(0 ; \lambda+\delta) & =0, \quad l=0,1, \ldots, n-2, \\
\bar{K}_{n}^{(l)}(0 ; \lambda) & =0, \quad l=1,2, \ldots, n-1 .
\end{aligned}
$$

Then for $l=1,2, \ldots, n-1$, taking the $l$-th derivative of both sides of $(6.9)$ with respect to $u$ yields

$$
\begin{aligned}
\frac{\partial^{l} \mathcal{L}(u, b)}{\partial u^{l}}= & \frac{(-1)^{l}}{c^{l+1}} \frac{\lambda^{n}}{(\lambda+\delta)^{n}} \int_{u}^{b} \zeta_{b}(s) k_{n}^{(l)}\left(\frac{s-u}{c} ; \lambda+\delta\right) d s \\
& +\left[e^{-\delta(b-u) / c} \bar{K}_{n}\left(\frac{b-u}{c} ; \lambda\right)\right]^{(l)}
\end{aligned}
$$

Setting $u=b$ in the above equation, and using (6.10) and (6.11), we obtain

$$
\left.\frac{\partial^{l} \mathcal{L}(u, b)}{\partial u^{l}}\right|_{u=b}=\left(\frac{\delta}{c}\right)^{l}, \quad l=1,2, \ldots, n-1 .
$$

This completes the proof. 
Theorem 6.4 $\mathcal{L}(u, b)$ satisfies the integro-differential equation

$$
\sum_{k=0}^{n} \frac{\partial^{k} \mathcal{L}(u, b)}{\partial u^{k}}\left[\frac{-(\lambda+\delta)}{c}\right]^{n-k}\left(\begin{array}{c}
n \\
n-k
\end{array}\right)=\left(\frac{-\lambda}{c}\right)^{n} \int_{0}^{u} \mathcal{L}(u-x, b) p(x) d x
$$

with boundary conditions given by Theorem 6.3.

Proof: To prove the integro-differential equation (6.12), for $j=1,2, \ldots$, $n-1$, we apply the operator $[\partial / \partial u-((\lambda+\delta) / c) \mathcal{I}]^{j}$ to both sides of $(6.9)$ and use $k_{n}^{\prime}(t ; \lambda+\delta)=(\lambda+\delta)\left[k_{n-1}(t ; \lambda+\delta)-k_{n}(t ; \lambda+\delta)\right]$. Then

$$
\begin{aligned}
& {\left[\frac{\partial}{\partial u}-\left(\frac{\lambda+\delta}{c}\right) \mathcal{I}\right]^{j} \mathcal{L}(u, b)} \\
& \quad=\frac{[-(\lambda+\delta)]^{j}}{c^{j+1}} \frac{\lambda^{n}}{(\lambda+\delta)^{n}} \int_{u}^{b} k_{n-j}\left(\frac{s-u}{c} ; \lambda+\delta\right) \zeta_{b}(s) d s \\
& \quad+\left(\frac{-\lambda}{c}\right)^{j} e^{-\delta(b-u) / c} \bar{K}_{n-j}\left(\frac{b-u}{c} ; \lambda\right)
\end{aligned}
$$

In particular, when $j=n-1$,

$$
\begin{aligned}
& {\left[\frac{\partial}{\partial u}-\left(\frac{\lambda+\delta}{c}\right) \mathcal{I}\right]^{n-1} \mathcal{L}(u, b)} \\
& =\frac{[-(\lambda+\delta)]^{n-1}}{c^{n}} \frac{\lambda^{n}}{(\lambda+\delta)^{n}} \int_{u}^{b}(\lambda+\delta) e^{-(\lambda+\delta)(s-u) / c} \zeta_{b}(s) d s \\
& \quad+\left(\frac{-\lambda}{c}\right)^{n-1} e^{-(\lambda+\delta)(b-u) / c}
\end{aligned}
$$

Applying the operator $[\partial / \partial u-((\lambda+\delta) / c) \mathcal{I}]$ again to both sides of $(6.14)$ and noting that $\zeta_{b}(u)=\int_{0}^{u} \mathcal{L}(u-x, b) p(x) d x$, we have

$$
\left[\frac{\partial}{\partial u}-\left(\frac{\lambda+\delta}{c}\right) \mathcal{I}\right]^{n} \mathcal{L}(u, b)=\left(\frac{-\lambda}{c}\right)^{n} \int_{0}^{u} \mathcal{L}(u-x, b) p(x) d x
$$

which is equivalent to the integro-differential equation (6.12).

We remark that the techniques presented in Section 3 can be applied to obtain explicit solutions for $\mathcal{L}(u, b)$.

\section{References}

[1] Dickson, D.C.M., 1998. On a class of renewal risk process. North American Actuarial Journal, 2(3), 60-68. 
[2] Dickson, D.C.M.,Hipp, C., 1998. Ruin probabilities for $\operatorname{Erlang(2)~risk~}$ process. Insurance: Mathematics \& Economics, 22, 251-262.

[3] Dickson, D.C.M., Waters, H.R., 2004. Some Optimal Dividends Problems. ASTIN Bulletin 34, 49-74.

[4] Gerber, H.U., Shiu, E.S.W., 2005. The time value of ruin in a Sparre Andersen model. North American Actuarial Journal, forthcoming.

[5] Grandell, J., 1991. Aspects of Risk Theory. Springer-Verlag, New York.

[6] Li, S., Garrido, J., 2004a. On ruin for $\operatorname{Erlang}(n)$ risk process. Insurance: Mathematics \& Economics, 34, 391-408.

[7] Li, S., Garrido, J., 2004b. On a class of renewal risk models with a constant dividend barrier. Insurance: Mathematics \& Economics, 35, 691701.

[8] Picard, P., 1994. On some measures of the severity of ruin in the classical Poisson model. Insurance: Mathematics \& Economics, 14, 107-115.

Shuanming Li and David C.M. Dickson

Centre for Actuarial Studies

Department of Economics

The University of Melbourne

Victoria 3010

Australia 


\section{University Library}

\section{- M M N E R VA A gateway to Melbourne's research publications}

Minerva Access is the Institutional Repository of The University of Melbourne

Author/s:

LI, S;DICKSON, D

Title:

The maximum surplus before ruin in an Erlang(n) risk process and related problems

Date:

2006

Citation:

LI, S. \& DICKSON, D. (2006). The maximum surplus before ruin in an Erlang(n) risk process and related problems. Insurance: Mathematics \& Economics, 38 (3), pp.529-539. https:// doi.org/10.1016/j.insmatheco.2005.11.005.

Persistent Link:

http://hdl.handle.net/11343/34358 